\title{
Diabetes and PKM2 affect prognosis in patients with intrahepatic cholangiocarcinoma
}

\author{
KUI FU ${ }^{1}$, XIAOLI YANG ${ }^{2}, \mathrm{HAO} \mathrm{WU}^{3}$, JIANPING GONG ${ }^{3}$ and XIUYUN LI ${ }^{1}$ \\ ${ }^{1}$ Department of Hepatobiliary Surgery, Traditional Chinese Medicine Hospital of Chongqing Dianjiang, \\ Chongqing 408300; ${ }^{2}$ Department of Hepatobiliary Surgery, Affiliated Hospital of Southwest Medical University, \\ Luzhou, Sichuan 646000; ${ }^{3}$ Department of Hepatobiliary Surgery, Second Affiliated Hospital of \\ Chongqing Medical University, Chongqing 400010, P.R. China
}

Received March 22, 2020; Accepted August 24, 2020

DOI: $10.3892 / \mathrm{ol} .2020 .12128$

\begin{abstract}
Diabetes mellitus (DM) is a common disease, but its effect on the prognosis of patients with intrahepatic cholangiocarcinoma (ICC) has not been reported. The aim of the present study was to explore the prognostic significance of diabetes in patients with ICC treated with hepatectomy and to clarify the role of pyruvate kinase M2 (PKM2). A consecutive retrospective cohort of 110 patients with ICC (28 with DM and 82 without DM) who underwent therapeutic hepatectomy was evaluated between January 2006 and January 2011. The clinicopathological characteristics of the two groups and the differences between overall survival (OS) and recurrence-free survival (RFS) were analyzed. The Cox proportional hazards model was further used to identify independent prognostic predictors. PKM2 expression was measured using immunohistochemical staining in tissues collected, after obtaining informed consent. Patients with ICC with DM exhibited significantly lower OS and RFS rates at 1, 3 and 5 years compared with patients with ICC without DM. Cox multivariate analysis revealed that DM was an independent predictor of poor OS and RFS. Additionally, high PKM2 expression was significantly higher in patients with ICC with DM compared with that in patients without DM. Overall, DM was associated with significantly lower OS and RFS rates in patients with ICC. The underlying biological rationale may be attributed to the higher PKM2 expression rate.
\end{abstract}

\section{Introduction}

Intrahepatic cholangiocarcinoma (ICC) is the second most common primary liver cancer, and its incidence is on the

Correspondence to: Dr Xiuyun Li, Department of Hepatobiliary Surgery, Traditional Chinese Medicine Hospital of Chongqing Dianjiang, 502 Gongnong Road, Chongqing 408300, P.R. China E-mail: cqdjlxy@163.com

Key words: diabetes mellitus, cholangiocarcinoma, pyruvate kinase M2, Warburg effect, glycolysis increase. It is a painful disease that poses a great burden to patients (1). Since ICC is resistant to radiotherapy and chemotherapy, surgical resection is considered to be the main treatment $(2,3)$. However, the prognosis in patients with ICC is poor, with an estimated median survival time of 18-39 months and a median 5-year survival rate of $25-40 \%(2,3)$.

Over the past 20 years, the global burden of diabetes mellitus (DM) has risen sharply and is expected to affect $>500$ million adults by 2030 , most of whom have type 2 diabetes (4). Previous studies have demonstrated that DM can significantly increase the risk of hepatocellular carcinoma (HCC) and ICC $(5,6)$. Some retrospective studies and meta-analyses identified DM as an independent predictor of poor prognosis in patients with HCC after hepatectomy (7-10). ICC and HCC originate from different cell types; however, obesity, diabetes, hepatitis $\mathrm{B}$ and $\mathrm{C}$, drinking and cirrhosis seem to be the main risk factors for both ICC and HCC, suggesting that ICC and HCC share a common pathogenesis mechanism (11). In addition, whether DM is also a prognostic factor of ICC after hepatectomy remains unclear.

Regardless of whether the supply of oxygen is sufficient, rapidly dividing cells convert glucose into lactate to produce ATP, which is known as aerobic glycolysis or the Warburg effect $(12,13)$. The Warburg effect is a widely observed feature of human cancer; in numerous types of tumor, including HCC and ICC, the presence of the Warburg effect is often associated with tumor invasiveness and a poor prognosis (14-16). The key rate limiting enzyme of the Warburg effect is pyruvate kinase M2 (PKM2), a tumor-specific subtype of pyruvate kinase, which catalyzes the synthesis of pyruvate and ATP with phosphoenolpyruvate and ADP as substrates $(17,18)$. Compensatory hyperinsulinemia is an important feature of type $2 \mathrm{DM}$ and is one of the upstream regulators of PKM2 expression $(19,20)$. The effect of DM on PKM2 expression in patients with ICC remains unknown.

The present study performed a retrospective analysis of patients with ICC who underwent hepatectomy at the Second Affiliated Hospital of Chongqing Medical University (Chongqing, China). The aims were to determine whether DM affects the prognosis in patients with ICC after hepatectomy and to provide a scientific basis for further exploration of the specific molecular mechanism. 


\section{Materials and methods}

Patients and follow-up. A total of 157 patients who underwent hepatectomy for ICC were eligible for inclusion in the present study at the Second Affiliated Hospital of Chongqing Medical University between January 2006 and January 2011. ICC was confirmed by histopathology and the anatomic location of the tumor was determined by review of histopathology, radiology and operation notes. After review, 47 of the 157 patients with ICC were excluded (15 lacked integrated clinical data, 13 lacked prospectively collected follow-up data and 19 had insufficient tissue for investigation). The remaining 110 patients (mean age, 53 years; age range, $28-79$ years; 62 males and 48 females; 28 with type $2 \mathrm{DM}$ and 82 without DM) were enrolled in the study. Cancerous and paracancerous tissues were obtained for both the DM and non-DM groups. Paracancerous tissue was defined as normal liver tissue $>5 \mathrm{~cm}$ away from the tumor.

DM was diagnosed as a fasting plasma glucose level of $>7.0 \mathrm{mmol} / 1(126 \mathrm{mg} / \mathrm{dl})$, or a plasma glucose level of $>11.1 \mathrm{mmol} / 1(200 \mathrm{mg} / \mathrm{dl})$ at $2 \mathrm{~h}$ in a $75-\mathrm{g}$ oral glucose tolerance test, or typical DM symptoms (polydipsia, polyuria, overeating, emaciation, fatigue or obesity) together with a casual plasma glucose level of $>11.1 \mathrm{mmol} / 1(200 \mathrm{mg} / \mathrm{dl}$; the normal fasting plasma glucose level is 3.9-6.1 mmol/l) (21).

All the patients were followed up until death or the end of the study in December 2016, with a median follow-up time of 55 months. After hepatectomy, all the patients were followed up every 3 months for 1 year and every 6 months thereafter. Liver function (based on the levels of alanine aminotransferase, aspartate aminotransferase, total bilirubin and albumin), prothrombin time (PT), abdominal ultrasound, chest film and enhanced CT or MRI were collected to monitor the patients. Diagnosis of tumor recurrence (intrahepatic and extrahepatic recurrence) was based on typical imaging findings. Tumor tissues were collected immediately upon resection from patients with ICC, and were subsequently fixed and paraffin-embedded, as described below, for immunohistochemistry.

The present retrospective study was conducted in accordance with the Declaration of Helsinki 2013 edition and national and international guidelines, and was approved by the Ethical Review Committee of the Second Affiliated Hospital of Chongqing Medical University. Written informed consent was provided by all patients.

Immunohistochemical staining. The ICC tissues were fixed in $4 \%$ paraformaldehyde at room temperature for $24 \mathrm{~h}$ and embedded in paraffin. Immunohistochemical staining of paraffin sections was performed using a two-step protocol. Briefly, the ICC sections (3- $\mu \mathrm{m}$-thick) were deparaffinized in xylene I for $15 \mathrm{~min}$ and xylene II for $15 \mathrm{~min}$ at $37^{\circ} \mathrm{C}$, and rehydrated in a graded ethanol series $(100,95,80$ and $75 \%$ ethanol for $5 \mathrm{~min}$ each). Subsequently, antigen retrieval was performed in $10 \mathrm{mmol} / \mathrm{l}$ sodium citrate solution (pH 6.0) at $100^{\circ} \mathrm{C}$ for $15 \mathrm{~min}$, and the samples were cooled for $30 \mathrm{~min}$ at room temperature. Endogenous peroxidase activity was inhibited using $3 \%$ hydrogen peroxide for $30 \mathrm{~min}$ at $37^{\circ} \mathrm{C}$, and $5 \%$ goat serum (Origene Technologies, Inc.) was used to block non-specific binding for $15 \mathrm{~min}$ at $37^{\circ} \mathrm{C}$, followed by incubation with a primary rabbit monoclonal anti-PKM2 antibody (1:1,000; cat. no. ab137852; Abcam) at $4^{\circ} \mathrm{C}$ overnight. Subsequently, the sections were incubated with a secondary anti-rabbit biotin-labelled IgG antibody (1:100; cat. no. SAP-9100; OriGene Technologies, Inc.) at $37^{\circ} \mathrm{C}$ for $30 \mathrm{~min}$. After washing with PBS, the visualization signal was detected using 3,3'-diaminobenzidine (Boster Biological Technology) and counterstaining was performed using hematoxylin at room temperature for $5 \mathrm{sec}$. To evaluate PKM2 expression, the slides were assessed independently by two experienced pathologists with minimal interobserver variability. The slides were assessed using an orthotopic light microscope (magnification, x100; Zeiss AG).

Scoring systems for immunohistochemical staining. A semi-quantitative assessment method score was used. Scoring parameters included the staining intensity (range, 0-3; 0 , negative; 1 , weak; 2 , moderate; and 3 , strong) and the percentage of positive cells (range, $1-4$; 0 , negative or $\leq 5 \%$; $1,6-25 \% ; 2,26-50 \% ; 3,51-75 \%$; and 4, 76-100\%). The staining intensity was based on the color of the positive markers: Light yellow indicated weak staining, brown-yellow indicated moderate staining and dark brown indicated strong staining. The percentage of positive cells and the intensity scores were added to determine the final staining scores. A total score $<4$ was defined as low PKM2 expression, while a score $\geq 4$ was defined as high PKM2 expression.

Statistical analysis. Statistical analyses were performed using SPSS 22.0 (IBM Corp.). The primary endpoint of the present study was overall survival (OS) after hepatectomy. OS was recorded as the time from the disease diagnosis to death due to any cause. Tumor recurrence-free survival (RFS) was recorded as the time from tumor resection to tumor recurrence. The significance of intergroup differences in continuous data was assessed using an unpaired Student's t-test to analyze the difference of PKM2 expression between ICC patients with $\mathrm{DM}$ and without DM, while the significance of differences in categorical data was assessed using the $\chi^{2}$ test or Fisher's exact test (two-tailed). Survival analysis was performed using the Kaplan-Meier method and the log-rank test. Univariate and multivariate analyses were performed using the Cox proportional hazards model to identify independent prognostic factors (multivariate analysis was based on significant results from the univariate analysis). The TNM staging system (22) was used to determine the tumor stage in the analysis of the clinicopathological features and prognosis of patients. Categorical variables are expressed as frequencies (\%). The results of the survival analysis are described as hazard ratios (HRs) and 95\% CIs. P<0.05 (two-sided) was considered to indicate a statistically significant difference.

\section{Results}

Clinicopathological features of patients with and without DM. Between January 2006 and January 2011, 110 patients (28 with type $2 \mathrm{DM}$ and 82 without DM) were included in the present study. The baseline characteristics were similar between the two groups, except that patients in the DM group had a higher frequency of vascular invasion ( 46.4 vs. $23.2 \%$; $\mathrm{P}=0.019)$. There were no significant differences in sex, age, TNM stage, tumor diameter, R0 resection, differentiation degree, lymph 
Table I. Statistical differences of clinicopathological characteristics between patients with DM (n=28) and without DM (n=82).

\begin{tabular}{|c|c|c|c|}
\hline Variables & DM, n $(\%)$ & Non-DM, n (\%) & P-value \\
\hline Sex (male) & $13(46.4)$ & $49(59.8)$ & 0.220 \\
\hline Age ( $\geq 45$ years) & $14(50.0)$ & $49(59.8)$ & 0.368 \\
\hline TNM stage (I-II) & $5(17.9)$ & $21(25.6)$ & 0.404 \\
\hline Tumor diameter $(>5 \mathrm{~cm})$ & $19(67.9)$ & $51(62.2)$ & 0.591 \\
\hline R0 & $17(60.7)$ & $47(57.3)$ & 0.753 \\
\hline Differentiation & & & 0.263 \\
\hline Low & $1(3.6)$ & $12(14.6)$ & \\
\hline Moderate & $23(82.1)$ & $62(75.6)$ & \\
\hline High & $4(14.3)$ & $8(9.8)$ & \\
\hline Lymph node metastasis & $10(35.7)$ & $25(30.5)$ & 0.608 \\
\hline Intrahepatic metastasis & $8(28.6)$ & $15(18.3)$ & 0.248 \\
\hline Multiplicity & $14(50.0)$ & $30(36.6)$ & 0.211 \\
\hline Vascular invasion & $13(46.4)$ & $19(23.2)$ & $0.019^{\mathrm{a}}$ \\
\hline Alanine aminotransferase (>100 IU/l) & $2(7.1)$ & $15(18.3)$ & 0.229 \\
\hline Aspartate aminotransferase (>100 IU/l) & $5(17.9)$ & $18(22.0)$ & 0.646 \\
\hline Total bilirubin $(>34 \mu \mathrm{mol} / \mathrm{l})$ & $4(14.3)$ & $20(24.4)$ & 0.264 \\
\hline Albumin (>35 g/dl) & $23(82.1)$ & $72(87.8)$ & 0.451 \\
\hline Prothrombin time (>14 sec) & $2(7.1)$ & $3(3.7)$ & 0.600 \\
\hline
\end{tabular}

${ }^{\mathrm{a}} \mathrm{P}<0.05$. DM, diabetes mellitus; IU, international unit.

node metastasis, intrahepatic metastasis, multiplicity, total bilirubin, alanine aminotransferase, aspartate aminotransferase, albumin and PT (Table I).

Association of DM and non-DM with OS after hepatectomy in patients with ICC. At the end of the follow-up period, $61(55.5 \%)$ patients had died. The median follow-up time was 55 months for all patients. The OS rate of patients with DM was significantly worse than that of patients without DM $(\mathrm{P}=0.004$; Fig. 1). The 1-, 3- and 5-year OS rates for patients with DM were 42.6, 23.0 and $23.0 \%$, which were lower than $83.8,48.2$ and $41.5 \%$ in patients without DM, and the median survival time in patients with and without diabetes was 10 and 36 months, respectively (Fig. 1). As shown in Table II, univariate analysis revealed that advanced TNM stage (HR, 0.474; 95\% CI, 0.245-0.918; $\mathrm{P}=0.027$ ), $>5 \mathrm{~cm}$ tumor diameter (HR, 0.460; 95\% CI, 0.260-0.814; $\mathrm{P}=0.008$ ), $\mathrm{R} 1$ resection (HR, 0.515; 95\% CI, 0.310-0.857; P=0.011), lymph node metastasis (HR, 2.865; 95\% CI, 1.658-4.952; $\mathrm{P}<0.001$ ), intrahepatic metastasis (HR, 9.266; 95\% CI, 4.863-17.657; $\mathrm{P}<0.001$ ), multiplicity (HR, 6.125; 95\% CI, 3.482-10.774; $\mathrm{P}<0.001$ ), vascular invasion (HR, 5.875; 95\% CI, 3.252-10.614; $\mathrm{P}<0.001$ ), high PKM2 expression (HR, 1.984; 95\% CI, 1.177-3.344; $\mathrm{P}=0.010)$ and $\mathrm{DM}(\mathrm{HR}, 2.152 ; 95 \% \mathrm{CI}, 1.255-3.691 ; \mathrm{P}=0.005)$ were adverse prognostic factors that affected OS in patients with ICC. In addition, multivariate analysis identified the following factors as independent predictors for poor OS: DM (HR, 1.989; 95\% CI, 1.084-3.65; P=0.026), high PKM2 expression (HR, 1.364; 95\% CI, 1.048-2.948; $\mathrm{P}=0.007$ ), intrahepatic metastasis (HR, 2.826; 95\% CI, 1.288-6.021; $\mathrm{P}=0.010)$, multiplicity (HR, 4.004; 95\% CI, 1.923-8.336; $\mathrm{P}<0.001)$ and vascular invasion (HR, 3.187; 95\% CI, 1.516-6.701; $\mathrm{P}=0.002)$.

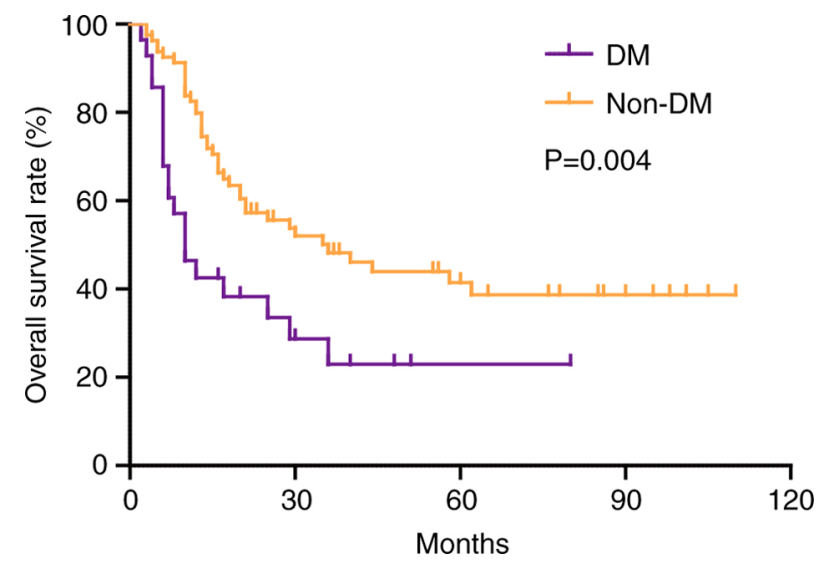

Figure 1. Kaplan-Meier survival curve analysis of overall survival rate in patients with intrahepatic cholangiocarcinoma according to DM. DM, diabetes mellitus.

Association of DM and non-DM with RFS after hepatectomy in patients with ICC. During follow-up, 78 (70.9\%) patients experienced tumor recurrence. The RFS rate in patients with DM was significantly worse than that in patients without DM ( $\mathrm{P}=0.004$; Fig. 2). The 1-, 3- and 5-year RFS rates for patients with DM were $32.1,16.3$ and $10.9 \%$, which were lower than $60.5,38.8$ and $31.7 \%$ in patients without DM, and the median RFS time in patients with and without diabetes was 5 and 17 months, respectively (Fig. 2). As shown in Table III, univariate analysis revealed that lymph node metastasis (HR, 2.664; 95\% CI, 1.638-4.331; P<0.001), intrahepatic metastasis (HR, 5.640; 95\% CI, 3.143-10.123; P<0.001), multiplicity (HR, 4.427; 95\% CI, 2.718-7.212; P<0.001), vascular 
Table II. Univariate and multivariate analysis of risk factors for overall survival.

\begin{tabular}{|c|c|c|c|c|c|c|}
\hline \multirow[b]{2}{*}{ Variables } & \multicolumn{3}{|c|}{ Univariate analysis } & \multicolumn{3}{|c|}{ Multivariate analysis } \\
\hline & HR & $95 \% \mathrm{CI}$ & P-value & HR & $95 \% \mathrm{CI}$ & P-value \\
\hline Sex (male/female) & 1.026 & 0.617-1.706 & 0.922 & & & \\
\hline Age $(<45 / \geq 45$ years $)$ & 0.850 & $0.506-1.428$ & 0.540 & & & \\
\hline TNM stage (I-II/III-IV) & 0.474 & $0.245-0.918$ & $0.027^{\mathrm{a}}$ & 1.003 & $0.462-2.176$ & 0.995 \\
\hline Tumor diameter $(\leq 5 />5 \mathrm{~cm})$ & 0.460 & $0.260-0.814$ & $0.008^{b}$ & 0.728 & $0.348-1.527$ & 0.402 \\
\hline R0 (R0/R1) & 0.515 & $0.310-0.857$ & $0.011^{\mathrm{a}}$ & 0.739 & $0.403-1.354$ & 0.327 \\
\hline Differentiation (low) & 1.000 & & & & & \\
\hline Differentiation (moderate) & 2.193 & $0.873-5.510$ & 0.095 & & & \\
\hline Differentiation (high) & 1.349 & $0.411-4.423$ & 0.622 & & & \\
\hline Lymph node metastasis (positive/negative) & 2.865 & $1.658-4.952$ & $<0.001^{\mathrm{c}}$ & 0.919 & $0.472-1.788$ & 0.803 \\
\hline Intrahepatic metastasis (positive/negative) & 9.266 & $4.863-17.657$ & $<0.001^{\mathrm{c}}$ & 2.826 & $1.288-6.201$ & $0.010^{\mathrm{a}}$ \\
\hline Multiplicity (positive/negative) & 6.125 & $3.482-10.774$ & $<0.001^{\mathrm{c}}$ & 4.004 & $1.923-8.336$ & $<0.001^{\mathrm{c}}$ \\
\hline Vascular invasion (positive/negative) & 5.875 & $3.252-10.614$ & $<0.001^{\mathrm{c}}$ & 3.187 & $1.516-6.701$ & $0.002^{\mathrm{b}}$ \\
\hline Pyruvate kinase M2 expression (high/low) & 1.984 & $1.177-3.344$ & $0.010^{\mathrm{a}}$ & 1.364 & $1.048-2.948$ & $0.007^{\mathrm{b}}$ \\
\hline Diabetes mellitus (yes/no) & 2.152 & $1.255-3.691$ & $0.005^{\mathrm{b}}$ & 1.989 & $1.084-3.650$ & $0.026^{\mathrm{a}}$ \\
\hline Total bilirubin $(>34 / \leq 34 \mu \mathrm{mol} / \mathrm{l})$ & 0.780 & $0.414-1.470$ & 0.442 & & & \\
\hline Alanine aminotransferase $(>100 / \leq 100 \mathrm{IU} / \mathrm{l})$ & 0.940 & $0.477-1.855$ & 0.859 & & & \\
\hline Prothrombin time $(>14 / \leq 14 \mathrm{sec})$ & 1.533 & $0.556-4.232$ & 0.409 & & & \\
\hline Albumin $(>35 / \leq 35 \mathrm{~g} / \mathrm{l})$ & 0.672 & $0.340-1.326$ & 0.252 & & & \\
\hline Aspartate aminotransferase $(>100 / \leq 100 \mathrm{IU} / \mathrm{l})$ & 1.310 & $0.731-2.348$ & 0.365 & & & \\
\hline
\end{tabular}

${ }^{\mathrm{a}} \mathrm{P}<0.05$; ${ }^{\mathrm{b}} \mathrm{P}<0.01$; ${ }^{\mathrm{C}}<0.001$. HR, hazard ratio; IU, international unit.

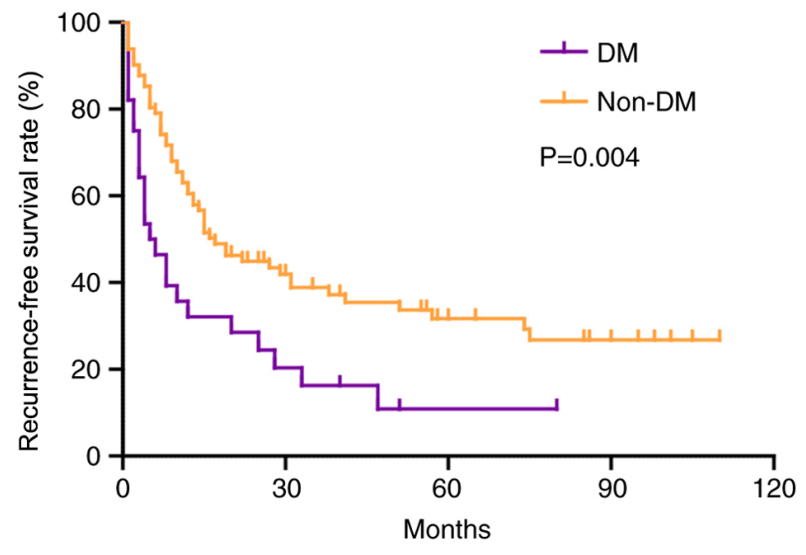

Figure 2. Kaplan-Meier survival curve analysis of recurrence-free survival rate in patients with intrahepatic cholangiocarcinoma according to DM. DM, diabetes mellitus.

invasion (HR, 5.100; 95\% CI, 3.017-8.619; $\mathrm{P}<0.001$ ), high PKM2 expression (HR, 2.048; 95\% CI, 1.289-3.254; $\mathrm{P}=0.002$ ) and DM (HR, 1.985; 95\% CI, 1.222-3.225; $\mathrm{P}=0.006)$ were adverse prognostic factors that affected RFS in patients with ICC. In addition, multivariate analysis identified the following factors as independent predictors for poor OS: DM (HR, 1.784; 95\% CI, 1.042-3.053; $\mathrm{P}=0.035)$, high $\mathrm{PKM} 2$ expression (HR, 1.567; 95\% CI, 1.057-3.012; $\mathrm{P}=0.006)$, multiplicity (HR, 2.898; 95\% CI, 1.564-5.369; $\mathrm{P}=0.001)$ and vascular invasion (HR, 2.655; 95\% CI, 1.340-5.259; $\mathrm{P}=0.005$ ).
Association of PKM2 expression in patients with ICC with and without DM. PKM2 expression was mainly concentrated in the cytoplasm and nucleus. Representative images of immunohistochemical staining are shown in Fig. 3A. High PKM2 expression was observed in 61 (55.5\%) patients with ICC; among these patients, $85.7 \%$ patients had DM, while $45.1 \%$ patients did not have DM (Fig. 3B). Similarly to HCC (23), high PKM2 expression was associated with poor OS and RFS (Fig. 3C and D). Notably, the combination of low PKM2 expression and no DM had a favorable prognostic value, while patients with high PKM2 expression and DM had the shortest OS ad RFS time (Fig. 4A and B).

\section{Discussion}

In the present retrospective study, it was revealed that DM was an independent prognostic factor for survival that significantly affected the OS and RFS rates of patients with ICC. The impact of DM was independent of patient demographics. In addition, patients with DM had a higher PKM2 expression rate than patients without DM, but the mechanism by which DM may regulate PKM2 expression remains to be uncovered. DM has been identified as an independent risk factor for ICC in a number of countries, and routine measurements for $\gamma$-glutamyl transferase and/or CA19-9 have been recommended during follow-up for DM to detect ICC at an early stage and expect a good OS (24); however, the effect of DM on the outcome of ICC has been rarely reported (24-27). A retrospective study by Endo et al (28) revealed that DM was a prognostic 
Table III. Univariate and multivariate analysis of risk factors for recurrence-free survival.

\begin{tabular}{|c|c|c|c|c|c|c|}
\hline \multirow[b]{2}{*}{ Variables } & \multicolumn{3}{|c|}{ Univariate analysis } & \multicolumn{3}{|c|}{ Multivariate analysis } \\
\hline & HR & $95 \% \mathrm{CI}$ & P-value & HR & $95 \% \mathrm{CI}$ & P-value \\
\hline Sex (male/female) & 0.922 & $0.591-1.440$ & 0.722 & & & \\
\hline Age $(<45 / \geq 45$ years $)$ & 1.058 & $0.675-1.660$ & 0.805 & & & \\
\hline TNM stage (I-II/III-IV) & 0.644 & $0.378-1.099$ & 0.107 & & & \\
\hline Tumor diameter $(\leq 5 />5 \mathrm{~cm})$ & 0.699 & $0.438-1.118$ & 0.135 & & & \\
\hline R0 (R0/R1) & 0.602 & $0.382-0.948$ & 0.028 & & & \\
\hline Differentiation (low) & 1.000 & & & & & \\
\hline Differentiation (moderate) & 2.052 & $0.938-4.489$ & 0.072 & & & \\
\hline Differentiation (high) & 1.355 & $0.491-3.742$ & 0.557 & & & \\
\hline Lymph node metastasis (positive/negative) & 2.664 & $1.638-4.331$ & $<0.001^{\mathrm{c}}$ & 1.065 & $0.582-1.948$ & 0.839 \\
\hline Intrahepatic metastasis (positive/negative) & 5.640 & $3.143-10.123$ & $<0.001^{\mathrm{c}}$ & 1.517 & $0.722-3.187$ & 0.271 \\
\hline Multiplicity (positive/negative) & 4.427 & $2.718-7.212$ & $<0.001^{\mathrm{c}}$ & 2.898 & $1.564-5.369$ & $0.001^{\mathrm{b}}$ \\
\hline Vascular invasion (positive/negative) & 5.100 & $3.017-8.619$ & $<0.001^{\mathrm{c}}$ & 2.655 & $1.340-5.259$ & $0.005^{\mathrm{b}}$ \\
\hline Pyruvate kinase M2 expression (high/low) & 2.048 & $1.289-3.254$ & $0.002^{\mathrm{b}}$ & 1.567 & $1.057-3.012$ & $0.006^{\mathrm{b}}$ \\
\hline Diabetes mellitus (yes/no) & 1.985 & $1.222-3.225$ & $0.006^{\mathrm{b}}$ & 1.784 & $1.042-3.053$ & $0.035^{\mathrm{a}}$ \\
\hline Total bilirubin $(>34 / \leq 34 \mu \mathrm{mol} / \mathrm{l})$ & 0.750 & $0.426-1.321$ & 0.319 & & & \\
\hline Alanine aminotransferase (>100/<100 IU/l) & 0.857 & $0.463-1.586$ & 0.623 & & & \\
\hline 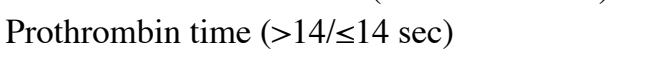 & 1.134 & $0.414-3.104$ & 0.807 & & & \\
\hline Albumin $(>35 / \leq 35 \mathrm{~g} / \mathrm{l})$ & 0.627 & $0.345-1.139$ & 0.126 & & & \\
\hline Aspartate aminotransferase (>100/ $\leq 100 \mathrm{IU} / \mathrm{l})$ & 1.198 & $0.706-2.032$ & 0.503 & & & \\
\hline
\end{tabular}

${ }^{\mathrm{a} P}<0.05 ;{ }^{\mathrm{b}} \mathrm{P}<0.01 ;{ }^{\mathrm{c}} \mathrm{P}<0.001$. HR, hazard ratio; IU, international unit.

A

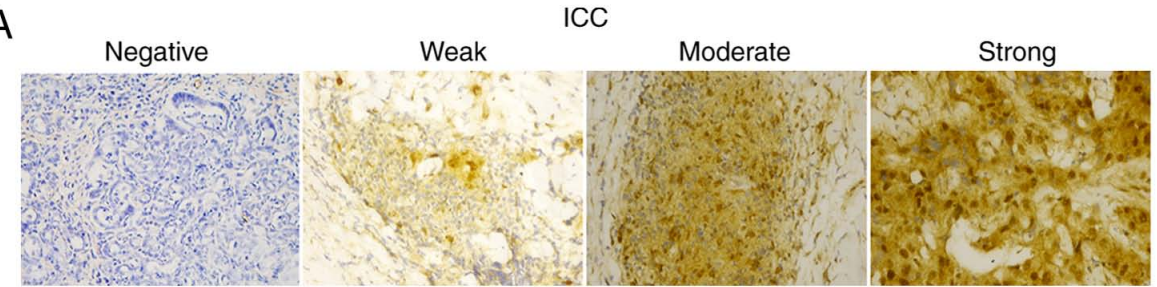

C

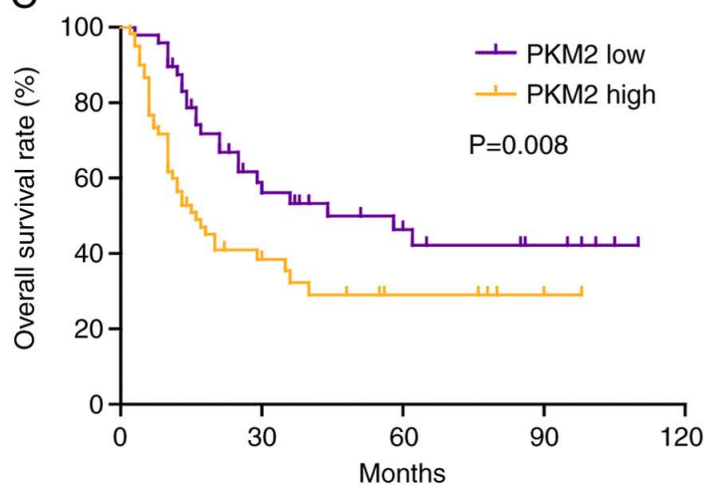

B

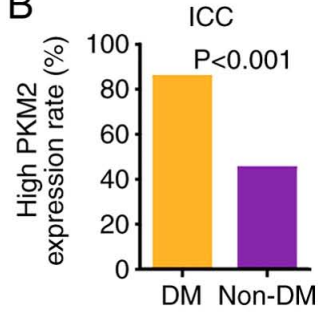

D

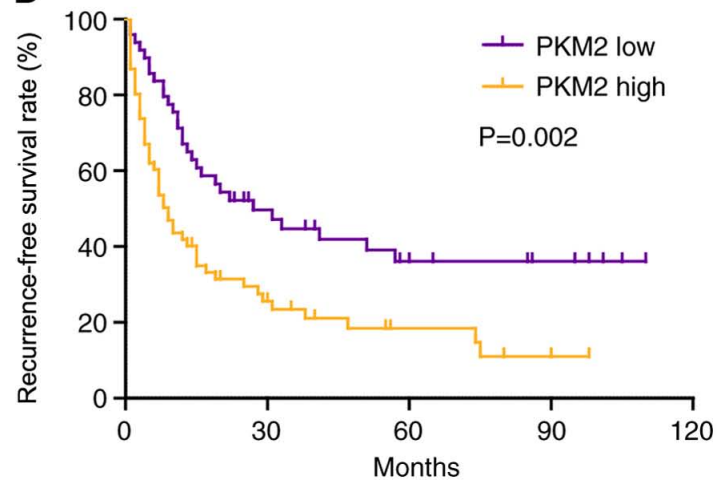

Figure 3. High PKM2 expression is associated with a poor prognosis in patients with ICC. (A) Immunohistochemical staining of PKM2 expression in ICC tissues. (B) Proportion of high PKM2 expression in patients with ICC with and without DM. Kaplan-Meier survival curve analysis of (C) overall survival rate and (D) recurrence-free survival rate in patients with ICC according to PKM2 expression. Magnification, x100. DM, diabetes mellitus; ICC, intrahepatic cholangiocarcinoma; PKM2, pyruvate kinase M2.

factor for patients undergoing surgery for ICC; however, only $6(6 / 81)$ patients with ICC had DM, affecting the reliability of the results. In the present cohort, $28(25.2 \%)$ patients had
DM, which is similar to a previously reported prevalence (4.9-33.1\%) in patients with ICC $(29,30)$. The current multivariate analysis revealed that DM was an independent risk factor 

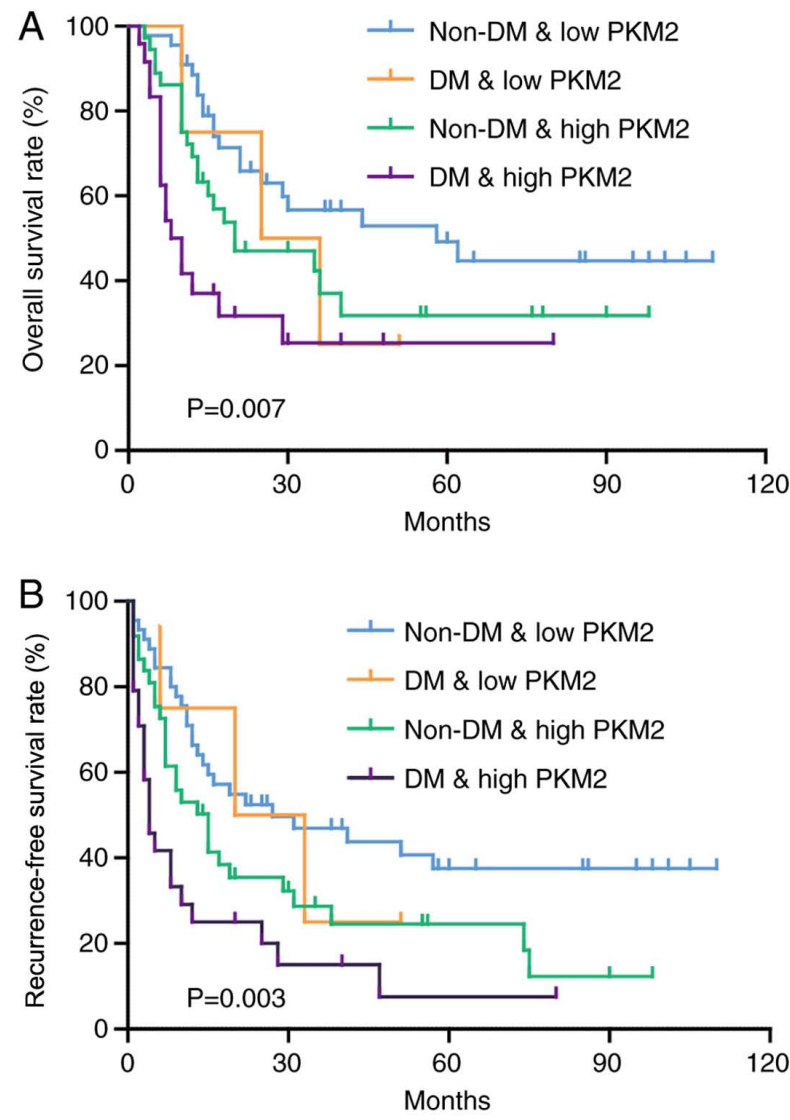

Figure 4. Patients with high PKM2 expression and DM have the shortest survival. (A) Kaplan-Meier survival curve analysis of overall survival rate (\%) in patients with ICC according to DM and PKM2 expression. (B) Kaplan-Meier survival curve analysis of Recurrence-free survival rate $(\%)$ in patients with ICC according to DM and PKM2 expression. DM, diabetes mellitus; ICC, intrahepatic cholangiocarcinoma; PKM2, pyruvate kinase $\mathrm{M} 2$.

for OS and RFS, suggesting that a more rigorous follow-up strategy should be adopted for patients with DM and suitable anti-diabetes treatments may be efficacious in patients with ICC complicated with DM.

PKM2 is widely expressed in cancer and can promote cancer cell proliferation through multiple biological mechanisms (31). In breast cancer, PKM2-Y105D phosphomimetic mutant increases MCF-10a cell colony formation and $\mathrm{CD}_{4} 4^{+} / \mathrm{CD} 24^{-}$cancer stem cell population by increasing YY1-associated protein 1 (YAP) nuclear localization (32). ErbB2 is a strong inducer of PKM2-Y105D phosphorylation, which promotes the nuclear localization of YAP and increases the number of tumor stem cells (32). PKM2 binds directly to histone $\mathrm{H} 3$ and phosphorylates histone $\mathrm{H} 3$ at threonine 11 when EGFR is activated; this phosphorylation is required for the separation of histone deacetylase 3 from cyclin D1 and Myc promoter regions, and subsequent acetylation of histone $\mathrm{H} 3$ at lysine 9 (33). PKM2-dependent histone $\mathrm{H} 3$ modification serves an important role in EGF-induced cyclin D1 and c-Myc expression, glioma cell proliferation, cell cycle progression and brain tumorigenesis (33). Further analysis of the mechanism of PKM2 revealed that mitomycin 2 (MFN2), a key regulator of mitochondrial fusion, interacted with PKM2, promoted mitochondrial fusion and production of phosphorus oxide, and suppressed glycolysis (34). Additionally, mTOR increases the interaction between PKM2 and MFN2 through phosphorylation of MFN2, and it regulates the effects of PKM2 and MFN2 on glycolysis, mitochondrial fusion and oxidative phosphorylation in hepatocellular carcinoma and lung cancer cells (34). Therefore, the mTOR-MFN2-PKM2 signal axis combines glycolysis with oxygen and phosphorus to regulate the growth of hepatocellular carcinoma and lung cancer cells (34). Insulin can increase PKM2 expression in vitro, but this has not been verified in the clinic $(19,20)$. To the best of our knowledge, the present data indicated for the first time in clinic samples that PKM2 expression was higher in patients with DM than in those without, consistent with previous in vitro results (23). Although the accurate underlying mechanism remains unknown, the current results may partially explain why patients with DM have a poorer survival outcome than patients without DM.

The present study presents some limitations. First, DM treatment can significantly affect long-term survival in patients with HCC (35). Therefore, the same phenomenon may be observed in patients with ICC. However, the DM treatment strategy in the present study was unknown. Second, all patients in the current cohort underwent surgical resection, but these patients represented only a small proportion of all patients with ICC (3). Whether DM also indicates a poor prognosis in patients without surgery remains to be explored.

In summary, the present data revealed that DM was associated with a significantly lower OS rate in patients with ICC. A potential cause may be associated with the abnormal glucose metabolism mediated by PKM2, which should be further investigated.

\section{Acknowledgements}

Not applicable.

\section{Funding}

The present study was supported by the National Natural Science Foundation of China (grant no. 81802778).

\section{Availability of data and materials}

All data generated and/or analyzed during this study are included in this published article.

\section{Authors' contributions}

KF, XY and HW wrote the manuscript. XY and HW were involved in the statistical analysis. XY, HW and JG were involved in clinical data collection. KF, XY, JG and $\mathrm{XL}$ were involved in the study design, acquisition of data, financial support and proofreading of the manuscript. All authors read and approved the final manuscript.

\section{Ethics approval and consent to participate}

All experiments were approved by the Ethics Committee of the Second Affiliated Hospital of Chongqing Medical University. Written informed consent was provided by all patients. 


\section{Patient consent for publication}

Not applicable.

\section{Competing interests}

The authors declare that they have no competing interests.

\section{References}

1. Shaib YH, Davila JA, McGlynn K and El-Serag HB: Rising incidence of intrahepatic cholangiocarcinoma in the United States: A true increase? J Hepatol 40: 472-477, 2004.

2. Spolverato G, Kim Y, Alexandrescu S, Popescu I, Marques HP, Aldrighetti L, Clark Gamblin T, Miura J, Maithel SK, Squires MH, et al: Is hepatic resection for large or multifocal intrahepatic cholangiocarcinoma justified? Results from a multi-institutional collaboration. Ann Surg Oncol 22: 2218-2225, 2015.

3. Raoof M, Dumitra S, Ituarte PHG, Melstrom L, Warner SG Fong Y and Singh G: Development and validation of a prognostic score for intrahepatic cholangiocarcinoma. JAMA Surg 152: e170117, 2017.

4. Whiting DR, Guariguata L, Weil C and Shaw J: IDF diabetes atlas: Global estimates of the prevalence of diabetes for 2011 and 2030. Diabetes Res Clin Pract 94: 311-321, 2011.

5. Yang WS, Shu XO, Gao J, Li HL, Cai H, Yang G, Ji BT, Rothman N, Gao YT, Zheng W and Xiang YB: Prospective evaluation of type 2 diabetes mellitus on the risk of primary liver cancer in Chinese men and women. Ann Oncol 24: 1679-1685, 2013.

6. Chaiteerakij R, Yang JD, Harmsen WS, Slettedahl SW, Mettler TA, Fredericksen ZS, Kim WR, Gores GJ, Roberts RO, Olson JE, et al: Risk factors for intrahepatic cholangiocarcinoma: Association between metformin use and reduced cancer risk. Hepatology 57: 648-655, 2013

7. Komura T, Mizukoshi E, Kita Y, Sakurai M, Takata Y, Arai K, Yamashita T, Ohta T, Shimizu K, Nakamoto Y, et al: Impact of diabetes on recurrence of hepatocellular carcinoma after surgical treatment in patients with viral hepatitis. Am J Gastroenterol 102: 1939-1946, 2007.

8. Ting CT, Chen RC, Chen CC, Liu MH, Chu D and Kuo NW: Diabetes worsens the surgical outcomes in cirrhotic patients with hepatocellular carcinoma. Tohoku J Exp Med 227: 73-81, 2012.

9. Wang YG, Wang P, Wang B, Fu ZJ, Zhao WJ and Yan SL: Diabetes mellitus and poorer prognosis in hepatocellular carcinoma: A systematic review and meta-analysis. PLoS One 9: e95485, 2014.

10. Wang YY, Huang S, Zhong JH, Ke Y, Guo Z, Liu JQ, Ma L, Li H, Ou BN and Li LQ: Impact of diabetes mellitus on the prognosis of patients with hepatocellular carcinoma after curative hepatectomy. PLoS One 9: e113858, 2014.

11. Palmer WC and Patel T: Are common factors involved in the pathogenesis of primary liver cancers? A meta-analysis of risk factors for intrahepatic cholangiocarcinoma. J Hepatol 57: 69-76, 2012.

12. Vander Heiden MG, Cantley LC and Thompson CB: Understanding the warburg effect: The metabolic requirements of cell proliferation. Science 324: 1029-1033, 2009.

13. Cairns RA, Harris IS and Mak TW: Regulation of cancer cell metabolism. Nat Rev Cancer 11: 85-95, 2011.

14. Yu G, Yu W, Jin G, Xu D, Chen Y, Xia T, Yu A, Fang W, Zhang X, $\mathrm{Li} Z$ and Xie K: PKM2 regulates neural invasion of and predicts poor prognosis for human hilar cholangiocarcinoma. Mol Cancer 14: 193, 2015.

15. Beyoglu D, Imbeaud S, Maurhofer O, Bioulac-Sage $\mathrm{P}$, Zucman-Rossi J, Dufour JF and Idle JR: Tissue metabolomics of hepatocellular carcinoma: Tumor energy metabolism and the role of transcriptomic classification. Hepatology 58: 229-238, 2013.

16. Beyoglu D and Idle JR: The metabolomic window into hepatobiliary disease. J Hepatol 59: 842-858, 2013.

17. Israelsen WJ, Dayton TL, Davidson SM, Fiske BP, Hosios AM, Bellinger G, Li J, Yu Y, Sasaki M, Horner JW, et al: PKM2 isoform-specific deletion reveals a differential requirement for pyruvate kinase in tumor cells. Cell 155: 397-409, 2013.
18. Cortes-Cros M, Hemmerlin C, Ferretti S, Zhang J, Gounarides JS, Yin H, Muller A, Haberkorn A, Chene P, Sellers WR and Hofmann F: M2 isoform of pyruvate kinase is dispensable for tumor maintenance and growth. Proc Natl Acad Sci USA 110 489-494, 2013.

19. Li Q, Liu X, Yin Y, Zheng JT, Jiang CF, Wang J, Shen H, Li CY, Wang M, Liu LZ and Jiang BH: Insulin regulates glucose consumption and lactate production through reactive oxygen species and pyruvate kinase M2. Oxid Med Cell Longev 2014: 504953, 2014

20. Li W, Wang J, Chen QD, Qian X, Li Q, Yin Y, Shi ZM, Wang L, Lin J, Liu LZ and Jiang BH: Insulin promotes glucose consumption via regulation of miR-99a/mTOR/PKM2 pathway. PLoS One 8: e64924, 2013.

21. American Diabetes Association: Standards of medical care in diabetes-2013. Diabetes Care 36 Suppl 1 (Suppl 1): S11-S66, 2013.

22. Edge SB, Byrd DR, Compton CC, Fritz AG, Greene FL and Trotti A (eds). AJCC Cancer Staging Manual. 7th edition. Springer, New York, NY, 2010

23. Liu Y, Wu H, Mei Y, Ding X, Yang X, Li C, Deng M and Gong J: Clinicopathological and prognostic significance of PKM2 protein expression in cirrhotic hepatocellular carcinoma and non-cirrhotic hepatocellular carcinoma. Sci Rep 7: 15294, 2017.

24. Nishioka T, Kubo S, Tanaka S, Wakasa K, Takemura S, Kinoshita M, Hamano G, Kuwae Y, Shibata T and Suehiro S: Outcomes of hepatic resection in intrahepatic cholangiocarcinoma patients with diabetes, hypertension, and dyslipidemia: Significance of routine follow-up. Liver Cancer 5: 107-120, 2016.

25. Lee BS, Park EC, Park SW, Nam CM and Roh J: Hepatitis B virus infection, diabetes mellitus, and their synergism for cholangiocarcinoma development: A case-control study in Korea. World J Gastroenterol 21: 502-510, 2015.

26. Wu Q, He XD, Yu L, Liu W and Tao LY: The metabolic syndrome and risk factors for biliary tract cancer: A case-control study in China. Asian Pac J Cancer Prev 13: 1963-1969, 2012.

27. Huang YJ, Wu AT, Chiou HY, Chuang MT, Meng TC, Chien LN and Yen Y: Interactive role of diabetes mellitus and female sex in the risk of cholangiocarcinoma: A population-based nested case-control study. Oncotarget 8: 6642-6651, 2017.

28. Endo I, Gonen M, Yopp AC, Dalal KM, Zhou Q, Klimstra D, D'Angelica M, DeMatteo RP, Fong Y, Schwartz L, et al: Intrahepatic cholangiocarcinoma: Rising frequency, improved survival, and determinants of outcome after resection. Ann Surg 248: 84-96, 2008.

29. Jing W, Jin G, Zhou X, Zhou Y, Zhang Y, Shao C, Liu R and $\mathrm{Hu} \mathrm{X}$ : Diabetes mellitus and increased risk of cholangiocarcinoma: A meta-analysis. Eur J Cancer Prev 21: 24-31, 2012.

30. Welzel TM, Graubard BI, El-Serag HB, Shaib YH, Hsing AW, Davila JA and McGlynn KA: Risk factors for intrahepatic and extrahepatic cholangiocarcinoma in the United States: A population-based case-control study. Clin Gastroenterol Hepatol 5: 1221-1228, 2007.

31. Dayton TL, Jacks T and Vander Heiden MG: PKM2, cancer metabolism, and the road ahead. EMBO Rep 17: 1721-1730, 2016.

32. Zhou Z, Li M, Zhang L, Zhao H, Sahin Ö, Chen J, Zhao JJ, Songyang Z and Yu D: Oncogenic kinase-induced PKM2 tyrosine 105 phosphorylation converts nononcogenic PKM2 to a tumor promoter and induces cancer stem-like cells. Cancer Res 78: 2248-2261, 2018.

33. Yang W, Xia Y, Hawke D, Li X, Liang J, Xing D, Aldape K, Hunter T, Alfred Yung WK and Lu Z: PKM2 phosphorylates histone $\mathrm{H} 3$ and promotes gene transcription and tumorigenesis. Cell 150: 685-696, 2012.

34. Li T, Han J, Jia L, Hu X, Chen L and Wang Y: PKM2 Coordinates glycolysis with mitochondrial fusion and oxidative phosphorylation. Protein Cell 10: 583-594, 2019.

35. Casadei Gardini A, Faloppi L, De Matteis S, Foschi FG, Silvestris N, Tovoli F, Palmieri V, Marisi G, Brunetti O, Vespasiani-Gentilucci U, et al: Metformin and insulin impact on clinical outcome in patients with advanced hepatocellular carcinoma receiving sorafenib: Validation study and biological rationale. Eur J Cancer 86: 106-114, 2017.

This work is licensed under a Creative Commons Attribution-NonCommercial-NoDerivatives 4.0 International (CC BY-NC-ND 4.0) License. 